\title{
La investigación cualitativa y el desarrollo profesional de enfermería
}

\author{
Judith Selene León Rivera, ,,2,a,b
}

León RJS. La investigación cualitativa y el desarrollo profesional de enfermería. Cuid salud, jul-dic 2015; 2(2):220-234.

\section{RESUMEN}

El campo del conocimiento es amplio y existen diversas formas de accederlo; la investigación científica y sus métodos son una forma de incrementar y llenar los vacíos en el conocimiento, siendo necesario para consolidar las bases epistemológicas de una profesión partiendo de la praxis. Este ensayo analiza brevemente el desarrollo de la investigación cualitativa, reconociendo su aporte a la comprensión del cuidado profesional de Enfermería, que se fundamenta en las experiencias de la persona dentro de un contexto particular, además de describir una aproximación del desarrollo de la investigación cualitativa en enfermería en el contexto peruano.

Palabras clave: investigación cualitativa, desarrollo profesional, enfermería, cuidado de enfermería.
León RJS. Qualitative research and professional development of nursing. Cuid salud, jul-dic 2015; 2(2):220-234.

\begin{abstract}
The field of knowledge is wide and there are different ways of accessing it; Scientific research and its methods are a way of increasing and filling the gaps in knowledge, being necessary to consolidate the epistemological basis of a profession starting from praxis. This essay briefly analyzes the development of qualitative research, by the recognition of its contribution to the understanding of professional nursing care, which is based on the experiences of the person within a particular context, apart from describing an approach of qualitative research development in nursing in the Peruvian context.
\end{abstract}

Keywords: Qualitative research, professional development, nursing, nursing care.

\footnotetext{
Enfermera Asistencial del Hospital Central de la Policía Nacional del Perú.

Universidad Privada San Juan Bautista-UPSJB.

Magíster en Enfermería.

b Doctoranda en Enfermería por la Universidad Nacional Mayor de San Marcos-Lima, Perú.
} 


\section{INTRODUCCIÓN}

Las bases que sustentan una profesión son los conocimientos, los cuales se generan y desarrollan a través de la investigación, siendo la universidad el espacio por excelencia, dónde se promueven eventos, se socializan y discuten los nuevos saberes para insertarlos luego en la práctica profesional.

Para Murrain, ${ }^{1}$ investigar supone aplicar la inteligencia a la exacta comprensión de la realidad objetiva a fin de dominarla; de ese modo será posible captar la esencia de las cosas y confrontarla con la realidad. Esta labor incrementa los conocimientos científicos de las disciplinas dando claridad, identidad y competencia al profesional.

A lo largo de la historia surgen diversos paradigmas filosóficos-científicos que sostienen el desarrollo de la ciencia. Guardián-Fernández ${ }^{2}$ define al paradigma como un sistema teórico dominante en cada período histórico de la ciencia, que organiza y dirige la investigación científica en una cierta dirección, sugiriendo hipótesis y centrando la atención del investigador en determinados aspectos del tema en estudio. Para Vasilachis, ${ }^{3}$ el paradigma es el marco teórico y metodológico que utiliza el investigador para el estudio de los fenómenos sociales en el contexto de una sociedad; destaca, además, tres paradigmas que permiten el acercamiento a la realidad: materialismo histórico, positivista e interpretativo.

El paradigma del materialismo histórico, propuesta por Marx, sustenta que el hombre es productor de sus representaciones, que su fuerza productiva, sus relaciones de producción, su entorno y sus interacciones lo convierten en lo que es en un momento histórico e influye en su modo de vida y que ésta a su vez determina la conciencia y no la conciencia a la vida. Asimismo, que los fenómenos sociales son transformados por el hombre, condicionando el proceso de la vida social, política, espiritual y por lo tanto, la evolución de una sociedad. ${ }^{4,5}$

Más adelante, en el primer tercio del siglo XIX surge el paradigma positivista. De acuerdo con Meza, ${ }^{6}$ sus inicios se le atribuyen al filósofo Francés Auguste Comte quien afirmaba que el único conocimiento verdadero es aquel que se deriva de la ciencia, en consecuencia, el positivismo asume que sólo las ciencias empíricas son fuente aceptable de conocimiento. Este paradigma sustenta la investigación cuantitativa, cuyos estudios parten de la identificación de las variables que están causando un fenómeno y su propósito es analizarlos, describirlos, asociarlos o explicarlos; de tal forma que explica por qué está ocurriendo ese fenómeno.

Con el paso del tiempo, los investigadores perciben que un hecho observable también tiene sentido y es posible comprenderlo a través de la experiencia humana, social y cambiante. GuardiánFernández $^{2}$ refiere que el surgimiento de paradigmas que involucran lo personal, lo ambiental, lo cualitativo y los derechos culturales reclaman su espacio vital, dando lugar al paradigma interpretativo; visión que se focaliza en la comprensión de lo humano que deriva de la inter-subjetividad y la interdisciplinariedad. Emergiendo por encima del positivismo, orientado hacia aspectos simbólicos y significativos de la vida social y del lenguaje en la producción y reproducción del mundo, cuyo objetivo es la captación y construcción de significados.

El paradigma interpretativo constituye una de las formas de investigación cualitativa; admite que los hechos, fenómenos o sucesos pueden registrarse narrativamente usando técnicas conocidas como la observación participante y las entrevistas no estructuradas, para luego ser interpretadas bajo un referencial teórico-filosófico. La diferencia fundamental entre el paradigma positivista e interpretativo es que la primera estudia la asociación o relación entre los fenómenos (variables) cuantificados buscando causa-efecto y, la segunda estudia los fenómenos en sus contextos estructurales y situacionales.

Bajo esta premisa, este ensayo tiene como objetivo analizar brevemente el desarrollo de la investigación cualitativa reconociendo su aporte a la comprensión del cuidado profesional de enfermería, que se fundamentan en las experiencias de la persona dentro de un contexto particular, además de describir una aproximación del desarrollo de la investigación cualitativa en enfermería en el contexto peruano.

\section{LA INVESTIGACIÓN CUALITATIVA}

Para comprender la realidad es sabio reconocer que no solo existe un camino, sino diversas posibilidades mediante el uso de supuestos filosóficos, intelectuales y disciplinarios. Desde finales del siglo XX se discute ampliamente sobre la polémica entre lo cualitativo-cuantitativo con reformulaciones constantes; al respecto Tójar ${ }^{7}$ señala que la historia de la ciencia y el 
conocimiento se ha desarrollado en base a la interacción y comunicación entre científicos y autores de los diversos campos del conocimiento, quienes comparten las mismas intenciones y conciben el mundo de manera muy similar, pero reconoce además, la existencia de una gran diversidad metodológica que permite la aproximación a los fenómenos.

El abordaje de la investigación depende de la intencionalidad y el enfoque que más se aproxime al objeto en estudio, trabajando con el universo de significados desde el punto de vista del investigado basado en su experiencia y vivencia. Para Sandoval, ${ }^{8}$ las investigaciones cualitativas reivindican el abordaje de las realidades subjetiva e intersubjetiva como objetos legítimos del conocimiento científico:

\section{"[...] el estudio de la vida cotidiana como \\ el escenario básico de construcción, constitución y desarrollo de los distintos planos que configuran e integran las dimensiones específicas del mundo humano, ponen de relieve el carácter único, multifacético y dinámico de las realidades humanas. Por esta vía emerge, entonces, la necesidad de ocuparse de problemas como la libertad, la moralidad y la significación de las acciones humanas dentro de un proceso de construcción socio-cultural $e$ histórico, cuya comprensión es clave para acceder a un conocimiento pertinente $y$ válido de lo humano" p.15}

De este modo comprender a la persona significa abordarla dentro de su anclaje socio cultural. Según Ariza, ${ }^{9}$ la investigación cualitativa nace en los años 20 y 30, cuando antropólogos y sociólogos la utilizaron para estudiar fenómenos humanos desde una visión holística, en el auge de las ciencias sociales.

Polit y Hungler ${ }^{10}$ refieren que la investigación cualitativa investiga un fenómeno particular, usualmente a profundidad y de forma holística mediante la recolección de información narrativa valiosa usando un diseño de investigación flexible. Field y Morse $^{11}$ acotan que los métodos de investigación cualitativa son útiles cuando se describe el fenómeno desde la perspectiva EMIC, que significa abordar el mundo subjetivo del sujeto desde su experiencia, interpretación y significado que le otorga a un evento. De acuerdo con Morse, ${ }^{12}$ el método de la investigación cualitativa puede ser caracterizado en tres aspectos: la perspectiva $E M I C$, la perspectiva holística y los procesos inductivos e interactivos de investigación. Esta autora concibe la perspectiva EMIC como extractora de significados, experiencias y percepciones desde el punto de vista del participante frente a aquella del investigador o la perspectiva dominante en el mundo.

Pita y Pértegas ${ }^{13}$ señalan que la investigación cualitativa trata de identificar la naturaleza profunda de las realidades, su sistema de relaciones y estructura dinámica de la experiencia cotidiana, poniendo énfasis en la subjetividad. Es decir, se interesa por cómo las experiencias son vividas e interpretadas por quienes las viven directamente.

Según Weber, citado por Rico, ${ }^{14}$ el mundo no puede deducirse solo por los significados otorgados a una acción, sino que se construyen intersubjetivamente en una realidad concreta y vivida buscando su comprensión y dándole sentido desde la mirada del otro. Weber la definió como Verstehen (comprensión, en alemán), que significa ir más allá del procedimiento de análisis lógico de las relaciones funcionales, es decir, encaminarse hacia la comprensión de las acciones y hasta de las intenciones de los sujetos.

\section{EL PARADIGMA INTERPRETATIVO}

Se entiende por paradigma al "modelo" o "arquetipo" de la concepción y estructura que crea el hombre para mirar su mundo y el mundo que le rodea; constituye una "visión de mundo", de cómo aprehende, percibe, comprende, explica y argumenta la naturaleza humana y sus construcciones. Para Kuhn: ${ }^{15}$

"[...] los paradigmas científicos son ejemplos aceptados de la práctica científica actual, ejemplos que combinan ley, teoría, aplicación e instrumentación y proporcionan modelos a partir de los cuales se manifiestan las tradiciones coherentes particulares de la investigación cientifica". p.10

Sandoval, ${ }^{8}$ citando a Kuhn, refiere que el paradigma como modelo constituye una totalidad compuesta por una concepción antropológica, filosófica y científico-metodológica, es decir, que existen múltiples realidades construidas por los actores relacionados con su entorno y realidad social. Realidad que emerge como una configuración de los diversos significados que las personas le otorgan a las situaciones que experimentan; construida a partir de sus marcos de referencia. 
El paradigma interpretativo es el más usado en estudios cualitativos, pues no pretende hacer generalizaciones a partir de sus resultados, sino más bien permitir la elaboración de una descripción ideográfica, individualizando al sujeto de estudio en sus múltiples interacciones para comprender los significados que le da a su propia conducta y a la conducta de los otros en su mundo de convivencia.

\section{CARACTERÍSTICAS DE LA INVESTIGACIÓN CUALITATIVA}

Taylor y $\operatorname{Bogdan}^{16}$ plantean que la investigación cualitativa, desde la investigación social, tiene las siguientes características: es inductiva porque su ruta conduce al descubrimiento y al hallazgo del fenómeno; holística porque mira a la persona desde la perspectiva de totalidad, su organización, su funcionamiento y su significación. Es interactiva y reflexiva porque requiere de una relación comunicativa y de intencionalidad; es naturalista y se centra en la lógica interna de la realidad dentro del marco de referencia de ellas mismas. La investigación cualitativa no impone visiones previas, más bien permite que el investigador se aleje de sus propias creencias, perspectivas y predisposiciones.
Se dice también que es abierta porque todos los escenarios y personas son dignos de estudio; es humanista, dado que se puede acceder por distintos medios a lo privado o personal como experiencias particulares, captado desde las percepciones, concepciones y actuaciones de quien los protagoniza. Finalmente, es rigurosa y busca resolver los problemas de validez y de confiabilidad por las vías de la exhaustividad (análisis detallado y profundo) y del consenso intersubjetivo (interpretación y sentidos compartidos). ${ }^{16}$

Martínez $^{17}$ considera que la investigación cualitativa es multimetódica por la variedad de datos e información que se obtiene a través de entrevistas, experiencia personal, historias de vida, rutinas, textos históricos, entre otros. Resalta que las orientaciones teóricas y el método usado ofrecen pautas específicas durante el desarrollo de la investigación. En la literatura se encuentran diversas formas de clasificar la investigación cualitativa: por tipos, métodos, técnicas $\mathrm{y}$ procedimientos. En este ensayo se reproduce la propuesta de Rodríguez et al. ${ }^{18}$ considerando la relación existente entre pregunta, método $\mathrm{y}$ técnicas de recolección de datos.

Tabla 1. Métodos y técnicas en investigación cualtitativa

\begin{tabular}{|c|c|c|c|}
\hline $\begin{array}{l}\text { Tipo de cuestiones a } \\
\text { Trabajar }\end{array}$ & Método & $\begin{array}{l}\text { Técnicas e instrumentos } \\
\text { de recogida de datos }\end{array}$ & $\begin{array}{l}\text { Otras fuentes de } \\
\text { Datos }\end{array}$ \\
\hline $\begin{array}{l}\text { Cuestiones de } \\
\text { significado, explicitar } \\
\text { la esencia de las } \\
\text { experiencias de los } \\
\text { actores }\end{array}$ & Fenomenología & $\begin{array}{l}\text { Grabación } \quad \text { de } \\
\text { conversaciones; escribir } \\
\text { anécdotas de experiencias } \\
\text { personales }\end{array}$ & $\begin{array}{l}\text { Literatura fenomenológica, } \\
\text { reflexiones filosóficas, poesía, } \\
\text { arte. }\end{array}$ \\
\hline $\begin{array}{l}\text { Cuestiones descriptivo } \\
\text { interpretativas: valores, } \\
\text { ideas prácticas de los } \\
\text { grupos culturales }\end{array}$ & Etnografía & $\begin{array}{l}\text { Entrevista no estructurada; } \\
\text { observación participante, } \\
\text { diagrama de redes } \\
\text { sociales }\end{array}$ & $\begin{array}{l}\text { Documentos, } \\
\text { registros, } \\
\text { fotografías, mapas, } \\
\text { genealogías, } \\
\text { diagramas de } \\
\text { redes sociales, } \\
\text { sociodramas }\end{array}$ \\
\hline $\begin{array}{l}\text { Cuestiones de proceso: } \\
\text { experiencia a lo largo } \\
\text { del tiempo o el cambio } \\
\text { puede tener etapas o } \\
\text { fases. }\end{array}$ & $\begin{array}{l}\text { Teoría } \\
\text { fundamentada }\end{array}$ & $\begin{array}{l}\text { Entrevistas } \\
\text { (grabadas) }\end{array}$ & $\begin{array}{l}\text { Observación } \\
\text { memorias, diarios. }\end{array}$ \\
\hline $\begin{array}{l}\text { Cuestiones centradas en } \\
\text { la interacción verbal y el } \\
\text { Diálogo }\end{array}$ & $\begin{array}{l}\text { Etnometodología; } \\
\text { análisis del } \\
\text { discurso }\end{array}$ & $\begin{array}{l}\text { Diálogo (registro en } \\
\text { audio y video) }\end{array}$ & Observación, notas de campo. \\
\hline $\begin{array}{l}\text { Cuestiones de mejora } \\
\text { y cambio social }\end{array}$ & Investigación acción & Diversos & Varios \\
\hline Cuestiones subjetivas & Biografía & Entrevista & $\begin{array}{l}\text { Documentos, } \\
\text { registros, diarios }\end{array}$ \\
\hline
\end{tabular}


La tabla 1 muestra diversas perspectivas epistemológicas adecuadas al interés del investigador y la naturaleza del fenómeno en estudio, especificando pautas especialmente al investigador novel en el paradigma interpretativo; permitiéndole sumergirse en cada realidad, captar y comprender el fenómeno desde la perspectiva del sujeto en sí mismo.

\section{LA INVESTIGACIÓN CUALITATIVA Y LA ENFERMERÍA}

En las últimas décadas, la profesión de enfermería atraviesa una serie de transformaciones derivado del avance de la ciencia, la tecnología y la generación de conocimientos que las enfermeras producen; influenciadas por la globalización, los cambios políticos, sociales, económicos y el perfil epidemiológico que exigen un cuidado de calidad.

Históricamente, la Enfermería nace como una práctica social basada en la experiencia del cuidado de la persona y su entorno. Posteriormente, desarrolla su propio cuerpo de conocimiento mediante el aporte de otras ciencias y de la investigación, con la finalidad de mejorar el cuidado de la persona en las distintas situaciones de salud, demostrando así que se requiere de una mirada diferente del sujeto, de quien debe considerar sus experiencias y necesidades, para luego ser analizados con carácter humanista y perspectiva integral.

Al considerarse una profesión que cuida a la persona en sus diferentes etapas de vida, sana o enferma, en el ámbito hospitalario o comunitario; tiene el objetivo de mantenerla en la mejor condición posible para conservar o recuperar su salud, reconociendo la importancia de su entorno, como fuera descrito por Florence Nightingale en 1860 y que cobra relevancia actualmente por la situación globalizada en que se vive. Amaro ${ }^{19}$ reconoce el rol pionero y protagónico de Nightingale en el desarrollo del pensamiento científico y ético de enfermería, partiendo de la importancia de la higiene, salud y su interrelación con la pobreza, dónde cobra relevancia el entorno. Pero destaca la necesidad de orientar el cuidado de enfermería hacia las preferencias del paciente y su autocuidado.

Cuesta, ${ }^{20}$ citando a Henderson, señala que para desarrollar investigación en enfermería el profesional debe colocarse en la subjetividad de la persona para poder identificar sus necesidades y trabajar con un método inductivo. Henderson refiere que el contacto con el paciente permite al profesional aproximarse a su experiencia humana y no a la enfermedad, situándose en su lugar para poder comprender su experiencia.

Los primeros trabajos cualitativos en enfermería surgen en el auge la sociología en la Escuela de Chicago, orientado hacia el trabajo de enfermería y sobre los estudiantes. ${ }^{20}$ Cuesta, $^{21}$ citando a Turner, refiere que los trabajos de enfermería realizado por los sociólogos datan de los años 50 con enfoque etnográfico, cuyos resultados muestran los conflictos de roles que había en ese entonces en enfermería, además de otros problemas cotidianos. En 1963, con el estudio de Davis y Olsen, citados por Murrain, ${ }^{1}$ se proponen las tres primeras líneas de investigación en enfermería visualizando el desafío que representaba el advenimiento del siglo XXI:

1) Los principios y leyes que gobiernan los procesos de la vida, bienestar y funcionamiento óptimo de los seres humanos enfermos o sanos;

2) Los patrones de comportamiento humano y su interacción con el medio ambiente en situaciones críticas de la vida, y

3) Los procesos por los cuales se afectan los campos positivos del estado de salud.

Líneas que actualmente se tornan relevantes, dado que el interés de enfermería es el cuidado integral del ser humano dentro de su contexto, entorno y realidad, donde la interacción es dinámica y al mismo tiempo compleja, aprendiendo a ser partícipe de su autocuidado en favor de su propia salud y de la salud de la comunidad con un todo.

Citando a Bottorff y Donaldson, Murrain ${ }^{1}$ reconoce que la investigación en enfermería es fuente de conocimiento que le otorga característica de disciplina, debiendo estar presente tanto en la práctica clínica como en el desarrollo de la administración y la educación, solo de ese modo se dará respuesta a las necesidades sociales que están relacionadas con procesos de salud-enfermedad, con las condiciones de vida y la calidad de vida de las personas, familias y comunidades, en todos los grupos etarios.

Ya entrando al nuevo milenio, el Consejo Internacional de Enfermería $(\mathrm{CIE})^{22}$ perfiló siete prioridades de investigación, con una clara tendencia hacia el enfoque cuantitativo, pues orienta la investigación hacia la búsqueda de 
causas, factores, calidad del cuidado e impacto de los programas e intervenciones de enfermería, tal y como se observa a seguir:

1) Generar conocimiento disciplinar relaciona con el desarrollo y aplicación de modelos conceptuales y teorías de enfermería;

2) Desarrollar estrategias para mejorar los programas de promoción de la salud y prevención de la enfermedad, en especial lo que corresponde a la determinación de factores de riesgo, incluyendo la vigilancia y control de los mismos;

3) Establecer características de las poblaciones y evaluar estrategias encaminadas a la modificación de factores de riesgo, puesto que se han dinamizado y transformado en los últimos años;

4) Analizar aspectos relacionados con la prestación de servicios de salud, sugiriendo mejorar la calidad del cuidado de enfermería y estudiar el impacto de sus intervenciones acorde con las metas de salud y bienestar;

5) Analizar la calidad de vida laboral de las enfermeras, la retención, movilidad local, regional e internacional, satisfacción laboral, estrés laboral, relación de enfermeras con la población, entre otros;

6) Participar en el desarrollo de políticas encaminadas a la equitativa, oportuna y eficiente prestación de servicios de salud, e

7) Integrar la práctica basada en la evidencia, lo cual dinamiza y amplía los horizontes de cuidado, puesto que surge desde escenarios distintos y promueve el trabajo en equipo entre la docencia y la asistencia.

Dichas líneas evidencian la necesidad de perfilar el hacer enfermero, partiendo de una realidad objetiva que describa qué hace enfermería, cómo hace y cómo está, elucidando el deber de promover su desarrollo desde un nuevo escenario para consolidar su identidad, con participación proactiva en el plano social y político del país, profundizando al mismo tiempo en la naturaleza esencial de la relación humana y compleja, realidad en la que emerge el enfoque cualitativo que motiva la exploración y el conocimiento de las personas con quienes se relaciona la enfermera y a partir de las cuales existe.

Leininger $^{23}$ realizó estudios utilizando métodos y técnicas cualitativas como la observación, documentación, análisis e interpretación de atributos, pautas, rasgos y significados de las características contextúales del cuidado, mostrando las particularidades antropológicas de algunas culturas. Para ella comprender el cuidado desde cada cultura es importante, así podría orientarse mejor el accionar, entendiendo las distintas maneras en cómo las personas previenen y se enfrentan a la enfermedad y sus varias formas de asumirla.

Vásquez, ${ }^{24}$ citando a Leininger, señala que cada persona, cada grupo o subgrupo tiene sus propias prácticas, creencias, valores y tradiciones. Por lo tanto, no es posible pretender homogenizar el cuidado y pensar que los de una cultura sean válidos para otra. Este aspecto es importante, sobre todo en el Perú, dónde la convergencia cultural se inició a consecuencia de problemas económicos y políticos a partir de los años 60 del siglo pasado, cambiando el perfil epidemiológico y más aún, el sistema de salud que dentro de sus políticas no considera el cuidado de la persona sino de la enfermedad. Realidad que hace imperativo una mirada holística e integral de las personas en cualquier contexto y situación, por ejemplo los métodos de investigación cualitativa importantes e impostergables, en el escenario local y nacional.

Entendiendo que la investigación cualitativa en enfermería facilita la observación y descripción de las respuestas humanas ante los procesos de salud-enfermedad, facultando a la enfermera a desplegar cuidados empáticos en cada cultura y contexto, es importante participar de las vivencias y experiencias conjuntas entre enfermera-paciente, acompañando de forma segura e interdependiente "un mundo nuevo" donde ambos crecen como parte de esa interrelación. La enfermera al captar y aprehender su mundo identifica sus necesidades y le ofrece al paciente mejores cuidados; además de comprender la humanidad y la integralidad de los demás.

\section{MÉTODOS CUALITATIVOS EN LA INVESTIGACIÓN DE ENFERMERÍA}

La investigación cualitativa posibilita una opción paralela para acceder al conocimiento y comprender la transformación de la realidad compleja que implica el cuidado de enfermería; la cual es integral, de calidad y está orientada a la persona independiente de su condición, donde es vital reconocer su problemas y, a su vez, buscar la práctica más adecuadas que garantice la conservación y mantenimiento de la salud en su entorno. 
Este reconocimiento de problema y solución se debe realizar a través de métodos que sean capaces de ayudar a interpretar y construir los significados que la persona otorga a sus experiencias y sobre esto proponer cuidados, tanto físicos como psicoespirituales, con el compromiso, comprensión y confianza que facilitará el empoderamiento de la persona para el autocuidado y el desarrollo humano profesional de la enfermera. A seguir se describen los métodos cualitativos más usados en enfermería según marco referencial:

1) Etnográfico, que considera importante la noción de cultura de la persona, dado que la interpretación y hallazgo que se describen suceden en un contexto específico; la observación participante y el trabajo de campo permiten obtener el conocimiento EMIC, donde el investigador no estudia a las personas, sino aprende de ellas y en base a ello realiza intervenciones favorables.

2) Teoría Fundamentada, la construcción de significado está basado en la utilidad que le otorgan las personas a los símbolos que usan diariamente a través de la interacción; dicho método ayuda a descubrir el significado que las personas construyen para explicar fenómenos de interés, resultando en la construcción de una teoría que está fundamentada en la información del investigado. Como estrategias para construir las categorías se sugiere preguntas a los datos, tales como: cuando ocurre, dónde ocurre, por qué ocurre, quién provoca, con cuáles consecuencias. Con esas simples preguntas se auxilia el proceso de construcción de categorías y fenómenos.

3) Fenomenología, se fundamenta en la experiencia vivida, posibilitando la mirada de las cosas tal como ellas se manifiestan; describiendo el fenómeno sin explicarlo, sin analizarlo; lo importante es la manera cómo las personas construye este fenómeno vivido a través de sus sentidos y el significado que le dan en el mundo donde vive, creando una perspectiva desde su propia experiencia, lo que nos ayuda a comprender experiencias humanas. En los estudios fenomenológicos se intentan ver las cosas desde el punto de vista de otra persona, describiendo, comprendiendo e interpretando su vivencia.

Dichos métodos permiten conocer a la persona $y$ sus experiencias frente a una enfermedad y el significado que le otorga desde su perspectiva; considerando que la búsqueda de la satisfacción de las necesidades humanas, la promoción de la salud y el empoderamiento de su autocuidado son áreas de interés de la profesión, favoreciendo la aceptación y participación de la persona en la recuperación y conservación de su salud.

En enfermería, múltiples estudios fueron realizados con enfoque cualitativo y métodos descriptivos, entre ellos el "Estudio sobre las tendencias de investigación sobre la práctica de enfermería en países de América Latina 19831987", 25 que plantea un análisis de la producción de enfermería considerando siete países, constituyendo el punto de partida para profundizar el conocimiento de la práctica de enfermería.

Prado et al. ${ }^{26}$ señalan que el desarrollo de investigaciones con metodología cualitativa en Brasil, aporta significativo conocimiento en el área asistencial, surgiendo principalmente de las perspectivas teóricas críticas en ciencias sociales y de las experiencias en estudios de investigaciónacción e investigación-acción participación; uno de los estudios identifica a la profesión de Enfermería como una práctica social donde el paciente, la enfermera y el equipo de Enfermería fueron percibidos como sujetos sociales, cuyo producto final consistía en atender las necesidades sociales $y$, en el caso de la salud, la recuperación de la persona o el control de la salud a nivel de la comunidad.

Similar situación se describe en el caso colombiano donde Nájera y Castrillón ${ }^{27}$ reportan que los estudios con enfoque cualitativo comienzan a desarrollarse en los 90, abordando la atención de enfermería en mujeres, niños y ancianos, a fin de superar la mirada empirista y biologisista de la construcción de conocimiento en la enfermería de ese entonces.

En Chile, Sahuenza ${ }^{28}$ afirma que el origen y desarrollo de la investigación cualitativa ha estado determinada por la contribución de los programas de postgrado con la formación de magíster y doctores en Enfermería, teniendo pendiente la aplicación de sus resultados en la práctica, junto con la consolidación de grupos de investigación para hacer investigación cualitativa en el ámbito de la disciplina, que permita la afirmación de la Enfermería como una disciplina social. 


\section{LA INVESTIGACIÓN EN LA ENFERMERÍA PERUANA: UNA APROXIMACIÓN A LA INVESTIGACIÓN CUALITATIVA}

La investigación científica en enfermería ha cobrado siempre un rol importante para el desarrollo de la profesión, contribuyendo en su consolidación como ciencia y evidencia de la práctica. Pero no es un camino fácil, diversas vicisitudes se enfrentan diariamente especialmente en la consolidación de las competencias investigativas.

Auccasi $^{29}$ señala que la Enfermería Peruana tuvo un lento proceso de cambio en el ámbito académico, asistencial, de investigación y administrativo, pues se consideraban aspectos de mayor complejidad las necesidades de los enfermos y los cuidados que requerían, además de la demanda de recursos humanos en los servicios de salud.

Aproximadamente en los años 90, existe un interés creciente de las enfermeras (principalmente del mundo académico) por mejorar sus condiciones académicas y laborales, se proponen y promueven cambios tendientes a la consolidación de la profesión; con la formación universitaria se promueve la creación de programas de especialización, maestría y doctorado, considerando que la educación de postgrado permite el dominio de la investigación, de la administración y de la docencia.

En lo referente a investigación, Auccasi ${ }^{29}$ opina que es su talón de Aquiles, identificando limitaciones en el manejo de proceso de investigación cualitativa. Sin embargo, reconoce que el profesional está interesado en ampliar su horizonte de conocimientos con apertura al nuevo mundo, accediendo a la realidad desde un enfoque más humano.

Zárate $^{30}$ señala que la investigación por esos años tuvo su mejor impulso en las aulas universitaria, principalmente en los programas de postgrado, cumpliendo un rol importante la Universidad Nacional de Trujillo, institución que a través de sus convenios internacionales muchas enfermeras peruanas se graduaron como Doctoras en Enfermería con el sólido compromiso de promover este nivel de formación en el país, paralelo a la organización de eventos científicos: congresos, coloquios y jornadas de investigación, a fin de discutir y socializar aspectos relevantes de la producción científica y su importancia en el campo profesional.
Asimismo, se promovió la publicación de artículos científicos a nivel nacional e internacional, además de la creación de varias revistas nacionales de enfermería, entre ellas Universidad Nacional de Trujillo, Universidad Peruana Cayetano Heredia, Colegio Nacional de Enfermeros, a fin de mostrar la producción científica que se venía produciendo y crear nuevos espacios y medios para difusión del conocimiento. Mismo así, esta difusión es limitada principalmente por parte del investigador, pero se perciben cambios e interés por generar más conocimientos, principalmente en el marco de la investigación cualitativa.

Según Neira, ${ }^{31}$ el ejercicio profesional de enfermería enfrenta numerosas responsabilidades; trabajo con jornadas extensas, gran número de pacientes, entorno laboral exigente, además de asuntos familiares y sociales. Sin embargo, señala que existe el deber profesional para desarrollar investigación científica, pues respalda su hacer cotidiano. La autora considera que la formación continua es un buen inicio para investigar y que el conocimiento adquirido en la formación universitaria que se adaptó a la práctica, debe volver a ser sistematizado.

Es cierto que la mayoría de investigaciones en enfermería se realizan bajo el enfoque cuantitativo, pero se reconoce la necesidad de emplear la metodología cualitativa que por sus características, permite comprender a la persona. Desarrollar y fortalecer conocimientos permite brindar un cuidado científico en la praxis, demostrando evidencia relevante y congruente con la realidad y con el desarrollo de la ciencia, a la vez analizar el contexto en que se vive y respetar la individualidad de cada persona, para aproximarse y conocer su mundo subjetivo, expectativas y necesidades, las cuales serán resueltas desde el accionar de enfermería. Además, estos conocimientos al ser divulgados y socializados permitirán mejorar la calidad del cuidado ofrecido a la persona sana o enferma.

De acuerdo con Mejía, ${ }^{32}$ en el investigador peruano, el método cualitativo toma real importancia en la década de los ochenta, donde existe un desarrollo progresivo en la sociología y las ciencias sociales, revalorizando al ser humano concreto como objeto central de análisis en contraste con las abstracciones y deshumanización del cientificismo positivista. Señala además, que el desarrollo de la metodología cualitativa es el 
resultado de los cambios que se operan en la investigación y en el objeto de estudio.

En el campo de enfermería la investigación cualitativa comienza en los estudios de doctorado, principalmente al iniciar el nuevo milenio en la Universidad Nacional de Trujillo, y posteriormente en la Universidad Nacional de San Marcos, dado que estos espacios tienen recurso humano capacitado, en su mayoría compuesto por profesionales que retornaron del hermano país de Brasil, o mediante sus profesores visitantes. En estos inicios se identifica un pluralismo metodológico que se va superando a los pocos; la mayoría de investigadores construye su referencial teórico-filosófico basado en las necesidades del fenómeno en estudio, sin necesariamente fundamentarlo en un referente filosófico metodológico. Estudios que se sustentan en la etnografía, las representaciones sociales parecen ser más fáciles de interpretar desde el desciframiento de los rasgos culturales y antropológicos. Otros abordajes como la fenomenología, la teoría de la complejidad, la etnometodología, parecen mucho más complejos y no siempre los resultados del estudio reflejan la esencia en sí mismo del referente filosóficometodológico.
La tabla 2 describe algunas investigaciones cualitativas realizadas en el país; adecuados a la clasificación propuesta por Rodríguez, ${ }^{18}$ reproduciendo el significado que los sujetos comprenden de su propia realidad, como los adultos mayores, estudiantes, internas de enfermería y profesionales asistenciales. Algunos se caracterizan como estudio de caso y en general, denominan "método de análisis" a la "trayectoria metodológica" del estudio, lo que evidencia aun el arraigo en el paradigma cuantitativo de los investigadores y sus mentores o, también puede reflejar las barreras que atraviesa la investigación cualitativa en el contexto peruano de la ciencias de la salud, donde aún es imperante el modelo biomédico empírico positivista.

Ínterin, cabe al investigador desarrollar cierta pericia y comprensión profunda del marco metodológico filosófico-sociológico usado como referente, para así poder develar lo que oculta el discurso y la expresión del sujeto en sí mismo. Existe mucho contenido en un lenguaje corporal, en las formas de dialogar y hablar; actitudes y comportamientos que no deben pasar desapercibidos por el investigador, de tal modo que pueda captar la verdadera realidad natural que busca ser develada en el estudio.

Tabla 2. Investigaciones cualitativas adecuadas a la clasificación de Rodríguez 1996

\begin{tabular}{|c|c|c|c|c|}
\hline Autor (es) & Local/Año & Titulo & Método & Consideraciones/conclusiones \\
\hline Vera $\mathrm{MM}^{33}$ & $\begin{array}{l}\text { UNMSM } \\
2007\end{array}$ & $\begin{array}{l}\text { Significado de la } \\
\text { calidad de vida } \\
\text { del adulto mayor } \\
\text { para sí mismo y } \\
\text { para su familia }\end{array}$ & $\begin{array}{l}\text { Interpretativo } \\
\text { Comprensivo }\end{array}$ & $\begin{array}{l}\text { Para el adulto mayor, la calidad de vida } \\
\text { significa tener paz y tranquilidad, ser } \\
\text { cuidado y protegido por la familia con } \\
\text { dignidad, amor y respeto, y tener } \\
\text { satisfechas como ser social sus necesidades } \\
\text { de libre expresión, decisión, comunicación } \\
\text { e información. Para la familia, que el adulto } \\
\text { mayor satisfaga sus necesidades de } \\
\text { alimentación, vestido, higiene y vivienda. }\end{array}$ \\
\hline $\begin{array}{l}\text { Verástegui } \\
\text { LR }^{34}\end{array}$ & $\begin{array}{l}\text { UNMSM } \\
2012\end{array}$ & $\begin{array}{l}\text { Significado del } \\
\text { cuidado de } \\
\text { enfermería } \\
\text { otorgado por las } \\
\text { enfermeras de un } \\
\text { servicio de cirugía } \\
\text { de un hospital de } \\
\text { Chiclayo } 2012\end{array}$ & $\begin{array}{l}\text { Representaciones } \\
\text { sociales }\end{array}$ & $\begin{array}{l}\text { Se fundamenta en cuatro dimensiones: } \\
\text { "Reconocimiento del ser cuidado como } \\
\text { persona humana en la interrelación", } \\
\text { "Reconocimiento de la propia naturaleza } \\
\text { humana de la enfermera", "Auto- } \\
\text { reconocimiento de capacidades de la } \\
\text { enfermera" y "Definiendo que el cuidado } \\
\text { de enfermería no es solo ciencia y arte, es la } \\
\text { complementación con el Self de la } \\
\text { enfermera". }\end{array}$ \\
\hline $\begin{array}{l}\text { Rentería } \\
\mathrm{VM}^{35}\end{array}$ & $\begin{array}{l}\text { Lambayeque } \\
2014\end{array}$ & $\begin{array}{l}\text { Cuidado } \\
\text { transcultural de la } \\
\text { enfermera } \\
\text { serumista en las } \\
\text { familias quechua } \\
\text { hablantes } \\
\text { Inkawasi-Perú }\end{array}$ & Etnográfico & $\begin{array}{l}\text { A pesar de las limitantes como el idioma, } \\
\text { pobreza extrema, distancias geográficas e } \\
\text { idiosincrasia de la población para cuidar de } \\
\text { su salud, la enfermera serumista se muestra } \\
\text { virtuosa al ser amable, sencilla y creativa, } \\
\text { siendo capaz de compartir momentos de } \\
\text { recreación y camaradería. Sin embargo, }\end{array}$ \\
\hline
\end{tabular}




\begin{tabular}{|c|c|c|c|c|}
\hline & & 2013 & & $\begin{array}{l}\text { existen cuidados que se pueden preservar, } \\
\text { negociar y reestructurar, para ello se } \\
\text { necesita utilizar estrategias como educación } \\
\text { intercultural, seguimiento, visitas } \\
\text { domiciliarias y alianzas estratégicas con } \\
\text { entidades públicas y privadas }\end{array}$ \\
\hline $\begin{array}{l}\text { Loli PA et } \\
\text { al. }{ }^{36}\end{array}$ & $\begin{array}{l}\text { UNMSM } \\
2015\end{array}$ & $\begin{array}{l}\text { La enseñanza } \\
\text { aprendizaje de la } \\
\text { investigación. } \\
\text { Representación } \\
\text { social desde la } \\
\text { perspectiva } \\
\text { estudiantil }\end{array}$ & $\begin{array}{l}\text { Representaciones } \\
\text { Sociales }\end{array}$ & $\begin{array}{l}\text { La representación social de la enseñanza } \\
\text { aprendizaje de la investigación en los } \\
\text { estudiantes se presentó como difícil, } \\
\text { compleja, estresante, relacionada con la } \\
\text { presión y controversias docentes, } \\
\text { sobrecarga de tareas y que lleva implícito } \\
\text { valores como la perseverancia, dedicación y } \\
\text { tiempo. Los momentos considerados como } \\
\text { más difíciles y trascendentes son el } \\
\text { planteamiento del problema, la } \\
\text { operacionalización de variables y la } \\
\text { sustentación. Se muestran actitudes } \\
\text { favorables a la investigación; sin embargo, } \\
\text { existe una dicotomía: "me gusta, pero } \\
\text { además, no tengo tiempo". }\end{array}$ \\
\hline $\begin{array}{l}\text { Cotos } \quad \mathrm{R}, \\
\text { Tejada } \mathrm{H}^{37}\end{array}$ & $\begin{array}{l}\text { Chimbote } \\
2014\end{array}$ & \begin{tabular}{l} 
Experiencias de la \\
persona adulta \\
hospitalizada \\
respecto al \\
cuidado brindado \\
por la enfermera \\
en el servicio de \\
cirugía \\
hospital del \\
ESSALUD De \\
\multicolumn{2}{l}{ Chimbote. Perú }
\end{tabular} & Fenomenología & $\begin{array}{l}\text { A través de la trayectoria fenomenológica } \\
\text { emergieron } 87 \text { unidades de significado, } \\
\text { sintetizadas en } 14 \text { categorías: resonancias } \\
\text { del cuidado, disonancias del cuidado, ser } \\
\text { cuidado: persona adulta hospitalizada, ser } \\
\text { enfermera: persona que brinda cuidados. El } \\
\text { cuidado puede ser percibido por la persona } \\
\text { hospitalizada como una forma de } \\
\text { experimentar un buen cuidado y trato } \\
\text { adecuado, atención oportuna a sus } \\
\text { necesidades; mientras que las divergencias } \\
\text { a estas percepciones como la inoportuna } \\
\text { atención de enfermería, experimentado por } \\
\text { la deficiente atención, que se ve reflejada } \\
\text { en errores en la comunicación e } \\
\text { información enfermera-paciente-familia. } \\
\text { Resulta necesario reflexionar sobre un } \\
\text { cuidado de enfermería altamente } \\
\text { humanizado, con un sólido componente } \\
\text { ético que permita crecer y desarrollar a la } \\
\text { enfermería como profesión. }\end{array}$ \\
\hline
\end{tabular}

En la tabla 2 los estudios muestran un proceso de construcción, donde las categorías alcanzadas no quedan claros, sobre todo en el último estudio, que menciona 14 categorías, que fueron agrupadas de acuerdo al fenómeno, pero no menciona como fueron agrupadas, además, en el análisis describe como es percibido el cuidado, considerando que la fenomenología busca develar el significado que le da una persona a su existencia en una determinada situación/condición. Para Castillo, ${ }^{38}$ la fenomenología hermenéutica se interesa en entender los fenómenos en sus propios términos, descubriendo y entendiendo los significados, hábitos y prácticas del ser humano en su mundo propio y como lo autointerpreta en el tiempo.

Lo que trae a colación la diversidad de métodos, técnicas y análisis de datos que se emplean para este tipo de investigación, evidenciándose diversidad, mixtura y en algunos casos incongruencia en la elaboración y desarrollo de los estudios, alentándose la idea de que realizar una investigación es difícil, sobre todo por la existencia de diversa y variada información, tomando difícil e incomprensible su manejo. Se destaca que la investigación debe desarrollarse de manera responsable, puesto que está al servicio del ser humano y el investigador asume las consecuencias del empleo de los métodos y de los resultados de sus investigaciones. ${ }^{39}$

Por esta situación también es vivida en otras realidades. Guardián-Fernandez, ${ }^{2}$ de Puerto Rico, en su papel de docente e investigadora, refiere que a pesar de la existencia de múltiples libros sobre investigación cualitativa, traducidos o escritos por autores de diferentes latitudes, otros contextos y 
otras visiones de mundo, de sociedad y de ser humano; desde el punto de vista epistémico, algunos estudios contradicen los fundamentos mismos de la investigación cualitativa. La autora cuestiona las posiciones hegemónicas de algunos y la confusión paradigmática de otros, la cual se evidencia al aproximarse al paradigma cualitativo desde una perspectiva eminentemente cuantitativa.

\begin{abstract}
Similar opinión tiene Salgado ${ }^{40}$ :
La metodología cualitativa ha abierto un espacio multidisciplinario que convoca a profesionales de las más diversas disciplinas[...] lo que lejos de ser un inconveniente aporta una gran riqueza en la producción. Sin embargo, también provoca una serie de efectos perversos, tales como, la gran variabilidad existente en la manera de afrontar el análisis: Imprecisión y confusión de conceptos, multiplicidad de métodos, más descripción que interpretación, riesgo de especulación, escasa visión de conjunto, ateorización, entre otros, hasta tal punto que hoy día no podríamos hablar del análisis cualitativo, sino más bien de los análisis cualitativos. El problema de acuerdo a algunos autores es que hay diversas visiones que se han considerado como tipos de investigación cualitativa y las bases epistemológicas son variadas. p.71
\end{abstract}

Es importante señalar que la comunidad científica y profesional están ávidas de conocimiento; aunque no siempre estén preparados para consumirlo, analizarlo y criticarlo; mismo así toda investigación debe ser documentada apropiadamente y estar en permanente difusión; creando espacios de discusión entre los profesionales de enfermería favoreciendo el desarrollo y la consolidación de la profesión con el aporte de nuevos conocimientos.

Se reconoce que existe aún mucho camino por recorrer y construir en la enfermería peruana, debiendo alentar y perseverar en la formación de noveles investigadores, que guiados por sus docentes investigadores conocedores del tema, encontraran asideros firmes para desarrollar investigación cualitativa, descubriendo con interés y creatividad su importancia y desarrollo; además, ofrecerán resultados sólidos y confiables que permitan brindar cuidados de calidad $y$ fundamentados en la realidad nacional. Reconociendo que una de las finalidades de la universidad es crear espacios permanentes de discusión y de motivación concreta de la investigación, contando con suficiente infraestructura como gabinetes, computadoras, redes de informática, bibliotecas con material actualizado y financiamiento concursable, que alienten y motiven el desarrollo de la investigación.

Es indudable que el camino suele ser largo; lo importante es haberlo iniciado, las mejoras se irán dando al construir la travesía, las historias se construyen con bajos y altos, con penas y glorias, con errores y aciertos; siendo importante saber mirar la propia realidad, develando incluso aquello que no es grato, pero que el enriquecimiento de la disciplina y la profesión de enfermería lo demandan, porque estos aspectos desalentadores son los que generan profunda reflexión y permiten visualizar el reto que se ambiciona.

\section{CONSIDERACIONES FINALES}

- A modo de consideración se puede decir que la investigación cualitativa busca la comprensión e interpretación de la realidad humana y social, con el propósito de conocer y orientar la acción y comprender la realidad subjetiva del sujeto de cuidado o dador de cuidado, dentro de su propio marco de referencia y contexto histórico-cultural, concibiendo la realidad tal como la viven, a partir de la interpretación de significados, sentimientos, creencias y valores. La investigación cualitativa brinda un sinfín de posibilidades para comprender a la persona sana o enferma que está bajo el cuidado de enfermería, así como su familia y entorno. La comprensión del ser humano dentro de su contexto ayuda a mejorar la práctica del cuidado, aplicar la metodología cualitativa permite explorar, develar y generar conocimiento de sus experiencias y significados.

- A nivel de Latinoamérica, el desarrollo de la investigación cualitativa en enfermería está siendo privilegiada, las instituciones académicas realizan ajustes a su plan de estudios, contando con docentes altamente capacitados e interesados en la formación de investigadores y en la constitución de núcleos de investigación, que permiten la discusión de temas sociales y de salud en favor de la construcción del conocimiento disciplinar. Experiencias exitosas constituyen Brasil, Colombia y Chile, donde el desarrollo del recurso humano de enfermería mediante capacitaciones, intercambio docenteestudiantil, financiamiento científico y 
tecnológico y, la difusión de su producción intelectual han hecho de estos países modelos a imitar. En el Perú la producción de conocimiento está ligada a la formación académica en pregrado y postgrado, siguiendo las iniciativas personales y del investigador senior; pocas investigaciones tienen la relevancia y el impacto esperado, fenómeno que se ve influenciado por múltiples factores, desde el proceso de aprendizaje del novel investigador, con escasa motivación interna y esporádicos espacios de socialización y difusión de conocimiento.

- Sobre la investigación cualitativa en Enfermería se evidencia dificultad en la búsqueda bibliográfica, en la selección y elección del marco referencial, poca claridad sobre los paradigmas de investigación, diferencias e incongruencias en el uso de métodos y el análisis datos, lo amerita un diagnóstico situacional para identificar las mayores dificultades e incidir en la elaboración de pautas que armonicen y guíen el desarrollo de estos estudios, iniciando así un proceso que conlleve al mejor entendimiento y desarrollo de la investigación cualitativa. Los métodos cualitativos son apropiados para realizar investigaciones en el campo de enfermería, sin

\section{Declaración de financiamiento y de conflictos de interés:}

El estudio fue financiado por el autor; declara no tener conflictos de interés.

\section{REFERENCIAS BIBLIOGRÁFICAS}

1. Murrain KE. Tendencias de la Investigación en Enfermería. Repert med cir [serie de internet]. 2009 [citado 15 de may 2015]; 18 (2):90-96. Disponible: http://repertorio.fucsalud.edu.co/pdf/vol18-022009/90-96.pdf

2. Guardián-Fernández A. El Paradigma cualitativo en la investigación socio-educativa [internet]. San José-Costa Rica: Coordinación Educativa y Cultural Centroamérica/Agencia Española de Cooperación Internacional; 2007 [citado 20 de may 2015]. 283p. Disponible en: https://web.ua.es/en/ice/documentos/recursos/ materiales/el-paradigma-cualitativo-en-lainvestigacion-socio-educativa.pdf embargo, es necesario establecer claramente las líneas de investigación que faciliten su desarrollo, además de la creación de grupos multicéntricos donde participen estudiantes de pre y postgrado, acompañados por docentes expertos en el área de investigación y expertos en el cuidado asistencial logrando la conjunción investigativa-asistencial; esto permitirá que los vacíos de conocimientos existentes en enfermería sobre el cuidado de paciente sean clarificados, trabajando en base a evidencias surgida de los estudios.

Finalmente, las instituciones educativas y los entes rectores de la profesión tienen la responsabilidad de desarrollar estrategias, proporcionar medios, normatizar y evaluar el rigor metodológico de las investigaciones cualitativas, participando activamente en el proceso de su elaboración, además de emitir opiniones expertas a través de una oficina de asesoría libre, unificando criterios y facilitando el entendimiento para el desarrollo del enfoque cualitativo.

\section{Correspondencia:}

Judith Selene León Rivera

Correo electrónico: judithselene@ hotmail.com

\section{Contribución de autoría:}

RGH, CMM, MSRT, FGS, HCH: Concepción y diseño del estudio, recolección de los datos, análisis e interpretación de los resultados y aprobación de la versión que será publicada.

3. Vasilachis I. El pensamiento de Habermas a la luz de una metodología propuesta de acceso a la teoría. Rev. Estudios Sociológicos [internet]. 1997 [citado 24 de may 2015]; 15(43): 1-30. Disponible en: http://estudiossociologicos.colmex.mx/index.p hp/es/article/download/874/874

4. Guerrero A. Manual de Sociología de la Educación. España ed. Síntesis 2002

5. García JC. Medicina y sociedad-Las corrientes de pensamiento en el campo de salud. Educación médica y salud. [internet] 1983 [citado 15 abril 2015]; 17(4), 363-397. Disponible:

http://www.unla.edu.ar/documentos/institutos/i sco/cedops/libro1a28.pdf 
6. Meza CLG. El paradigma positivista y la concepción dialéctica del conocimiento. Matemática, Educación e Internet [serie de internet]. 2003 [citado 20 de may 2015]; 4 (2): [aproximadamente 06 laudas]. Disponible en: http://revistas.tec.ac.cr/index.php/matematica/a $\underline{\text { rticle/view/2296 }}$

7. Tójar JC. Investigación Cualitativa: Comprender y Actuar [internet]. Madrid: Edit. La Muralla; 2006 [citado 28 de mar 2015]. 432 p. Disponible en: https://books.google.es/books/about/Investigaci ón_cualitativa.html?hl=es\&id

8. Sandoval CCA. Investigación Cualitativa [internet]. Colombia; 2002 [citado 14 de nov 2014]. 313 p. Disponible en: https://www.google.com.pe/webhp?sourceid=c hromeinstant\&rlz=1C1NHXL_esPE683PE683\&ion= $1 \&$ espv $=2 \&$ ie $=$ UTF -

9. Ariza OC. Aportes de la Investigación Cualitativa a la Enfermería. Investigación en Enfermería Imagen y Desarrollo [serie de internet]. 2005 [citado 15 de mar 2015]; 7(1,2): 8-13. Disponible en. http://revistas.javeriana.edu.co/index.php/imag enydesarrollo/article/view/1565/991

10. Polit D, Hungler B. Investigación científica en ciencias de la salud. $2^{\text {a }}$ ed. México: Interamericana; 1985.

11. Field PA, Morse JM. Qualitative research methods for health professionals [internet]. $2^{\mathrm{a}}$ ed. USA: Thousand Oaks; 1995 [citado 24 de may 2015]. Disponible en: https://books.google.es/books/download/Qualit ative Research Methods for Health.bibtex?id $=$ =PxZtAAAAMAAJ\&hl=es\&output=bibtex

12. Morse JM. Critical issues in qualitative research methods [internet]. USA: SAGE Publications; 1994. [citado 12 de jun 2014]. Disponible en: https://books.google.es/books/download/Critica 1 Issues in Qualitative Research.bibtex?id=3 ZlrPlpU10AC\&hl=es\&output=bibtex

13. Pita FS, Pértegas DS. Investigación cuantitativa y cualitativa. Unidad de Epidemiología Clínica y Bioestadística. Complexo Hospitalario Juan Canalejo. Cad Aten Primaria [serie de internet]. 2002 [citado el 23 may 2015]; 9: 76-78. Disponible en: http://prod.fisterra.com/mbe/investiga/cuanti_c uali/cuanti_cuali2.pdf

14. Rico GP. Elementos teóricos y metodológicos para la investigación educativa. Unidad 164. Zitácuaro-México: Universidad Pedagógica Nacional; 2005.

15. Kuhn T. La estructura de las revoluciones científicas. México, Fondo de Cultura Económica.1971

16. Taylor SJ, Bogdan R. Introducción a los métodos cualitativos de investigación. Barcelona: Paidós Básica, 1992.

17. Martínez VGL. Paradigmas de Investigación Manual multimedia para el desarrollo de trabajos de investigación. Una visión desde la epistemología dialéctico crítica [internet] 2013 [citado 16 de jun 2015]. 10 p. Disponible en: www.pics.uson.mx/wpcontent/uploads/.../7_Paradigmas de investiga cion_2013.pdf

18. Rodríguez G, Gil J, García E. Metodología de la investigación cualitativa [internet]. GranadaEspaña: Aljibe; 1996 [citado 7 de jul 2014]. 39p. Disponible en: http://metodosdeinvestigacioninterdisciplinaria. bligoo.com.co/media/users/10/528344/files/539 53/INVESTIGACION_CUALITATIVA_Rodri guez_et_al.pdf

19. Amaro CM. Florence Nightingale, la primera gran teórica de enfermería. Rev Cubana Enfermer [Internet]. 2004 [citado 03 de may de 2015]; 20(3): Disponible en: http://scielo.sld.cu/scielo.php?script=sci_arttext \&pid=S0864-03192004000300009\&lng=es

20. Cuesta BC. Características de la Investigación Cualitativa y su Relación con la Enfermería. Invest y Educac en Enfer [serie de internet]. Sep 1997 [citado 23 de oct 2014]; 15(2): 14$24 . \quad$ Disponible en: http://aprendeenlinea.udea.edu.co/revistas/inde x.php/iee/article/view/16941/14672

21. Cuesta BC. Investigación Cualitativa y Enfermería. Index de Enfermería [serie de internet] 2000 [citado 22 de may 2015]; 2829:7-8. Disponible en http://www.indexf.com/index-enfermeria/28-29revista/2829 articulo 7-8.php 
22. Consejo Internacional de Enfermería (CIE). La investigación en Enfermería: Instrumento de acción [Internet]. Ginebra: CIE; 2002 [citado 8 de jun 2015]: Disponible en: http://www.icn.ch/matters_researchsp.htm

23. Leininger MM. Cuidar a los que son de culturas diferentes requiere el conocimiento y las aptitudes de la enfermería transcultural (editorial). Cultura de los cuidados [serie de internet]; 1999 [citado 6 de mar 2015]; 3(6):5$9 . \quad$ Disponible en: https://rua.ua.es/dspace/bitstream/10045/5126/ 1/CC_06_01.pdf

24. Vásquez ML. El cuidado cultural adecuado: de la investigación a la práctica. Grupo de Cuidado Facultad de Enfermería Universidad Nacional de Bogotá: El arte y la ciencia del cuidado. Bogot-a-Colombia: Unibiblos; 2002. $120 \mathrm{p}$.

25. Sociedad Chilena de Enfermería. Estudio sobre las tendencias de investigación sobre la práctica de enfermería en países de América Latina (1983-1987): informe final de Chile. Ministerio de Salud. Chile 1987.

26. Prado DL, Souza ML, Carraro TE. Investigación cualitativa en enfermería, contexto y bases conceptuales. Mexico: OPS. Paltex 2008.

27. Nájera RM, Castrillón MCA. Aportes de los Coloquios Panamericanos a la investigación en enfermería. En Memorias del VII Coloquio Panamericano de Investigación en Enfermería. Santa Fe de Bogotá 2000.

28. Sanhueza AO. Proyección de la investigación cualitativa en enfermería en Chile. Rev. Cienc enferm [serie de internet]. Abr 2012 [citado 22 de ago 2015] 18 (1):7-8. Disponible en: http://www.scielo.cl/pdf/cienf/v18n1/art_01.pd $\underline{\mathrm{f}}$

29. Auccasi M. Evolución de Enfermería en el Perú en las Últimas Cinco Décadas del Siglo XX [internet] Perú. 1996. [citado 29 mar 2014] Disponible en: www.enfermeriaperu.com/eduenfer/evolenfer.h $\underline{\operatorname{tm}}$

30. Zárate M. De Escuela a Facultad Transición en la Educación de la Enfermería en el Perú.
Lima-Perú: Centro Editorial Universidad Peruana Cayetano Heredia; 2013. 284p.

31. Neira F. Enfermería y el ejercicio de la investigación. Ciencia y Arte de Enfermería [Internet]. 2015 [30 de mar 2015]; 1(1):4-5. Disponible en: http://www.cr3.org.pe/ojs/index.php/ace/article /download/11/12

32. Mejía J. Problemas Metodológicos de las Ciencias Sociales en el Perú. Fondo Editorial de la Facultad de Ciencias Sociales-UNMSM. 2002

33. Vera M. Significado de la calidad de vida del adulto mayor para sí mismo y para su familia. An Fac Med Lima, 2007; 68(3): 284-290.

34. Verástegui R. Significado del cuidado de enfermería otorgado por las enfermeras de un servicio de cirugía de un hospital de Chiclayo [tesis doctoral]. Lima: Universidad Nacional Mayor de San Marcos [Internet]; 2012 [citada 15 de may 2015]; 225p. Disponible en http://cybertesis.unmsm.edu.pe/handle/cybertes is $/ 3620$

35. Rentería MA. Cuidado transcultural de la enfermera serumista en las familias quechua hablantes Inkawasi-Perú [tesis de licenciatura]. Lambayeque: Universidad Católica Santo Toribio de Mogrovejo [internet]; 2014 [citada 22 de may 2015]; 125 p. Disponible en http://tesis.usat.edu.pe/jspui/handle/123456789 $\underline{1176}$

36. Loli PAR, Sandoval VMH, Ramírez ME, Quiroz VMF, Casquero NRA, Rivas DLH. La enseñanza aprendizaje de la investigación. Representación social desde la perspectiva estudiantil. An Fac Med, 2015; 76(1): 47-56.

37. Urquiaga AME. Vivencias de los profesionales de enfermería en la gestión del cuidado a personas hospitalizadas [tesis doctoral]. Chimbote: Universidad Católica Los Ángeles de Chimbote [Internet]; 2015 [citada 20 de jul 2015]; 90p. Disponible en: http://repositorio.uladech.edu.pe/handle/12345 $\underline{6789 / 74}$

38. Castillo EE. La fenomenología interpretativa como alternativa apropiada para estudiar los fenómenos humanos. Investigación y educación en enfermería [serie de internet]; 
2000 [citado 23 dic 2014]. 18(1): 27-35.

Disponible

en:

http://www.redalyc.org/articulo.oa?id=105218

$\underline{294002}$

39. Bernal TC, Correa PA, Pineda RM, Lemus HF, Fonseca YM, Muñoz RC. Fundamentos de Investigación. Mexico: Pearson Educación; 2014.

40. Salgado LAC. Investigación Cualitativa: diseños, evaluación del rigor metodológico y retos. Liber [serie de internet]. 2007 [citado el 16 mar 2015]; 13 (13):71-78. Disponible en: http://www.scielo.org.pe/pdf/liber/v13n13/a09 v13n13.pdf 